\title{
Análisis fotoelástico descriptivo de dos diferentes diseños de implantes endoóseos sometidos a carga de compresión vertical
}

\section{Descriptive photoelastical analysis of two different dental implants design submitted to vertical compression load}

\author{
JAIMES M* \\ DUQUE DE MIRANDA CHAVES NETTO H* \\ OLATE $S * *$ \\ MAZZONETTO R*** \\ ALBERGARIA BARBOSA JR***
}

\begin{abstract}
Jaimes M, Duque de Miranda Chaves Netto H, Olate S, Mazzonetto R, Albergaria Barbosa JR. Análisis fotoelástico descriptivo de dos diferentes diseños de implantes endoóseos sometidos a carga de compresión vertical. Av Periodon Implantol. 2009; 21, 3: 135-140.
\end{abstract}

\begin{abstract}
RESUMEN
El objetivo de este estudio fue analizar la distribución de las tensiones generadas en los modelos fotoelásticos posterior a la aplicación de cargas verticales a 7,5 N, en dos diferentes diseños de implantes endoóseos. Fueron seleccionados 2 implantes con diferentes configuraciones: Implante (Máster Conect Cônico ${ }^{\circledR}$ 4,13×13 mm) e Implante (Titamax Ti Medula ${ }^{\circledR} 3,75 \times 13 \mathrm{~mm}$ ). Para el análisis de la distribución de tensiones fueron confeccionados 2 modelos en resinas fotoelástica con dimensiones de $40 \times 10 \mathrm{~mm}$ de base y $30 \mathrm{~mm}$ de altura. Cada implante posicionado en los modelos recibieron carga vertical a 7,5 N, siendo posible visualizar las franjas isocromáticas generadas en los modelos, a través del polariscopio. El implante Máster Conect Cônico ${ }^{\circledR}$ con hexágono interno largo, porción cervical pulida, tope de las roscas redondeadas, pasos de las roscas continuas, distancia entre estas de 0,5 décimas de milímetros, espirales angulados y ápice cónico presento concentración de las tensiones en la región apical. El implante cilíndrico, Titamax Ti Medula ${ }^{\circledR}$, con hexágono externo, porción cervical pulida, con tope de las roscas en forma de $\mathrm{v}$, paso de rosca continuo, con distancia entre las roscas de 0.5 décimas de milímetros, espirales angulados y ápice redondeado presento distribución de las tensiones en la región apical medio y cervical del implante. Basado en los resultados de este estudio puede concluirse que: el implante de configuración cónica concentró las tensiones en región apical, siendo que el implante de configuración cilíndrica concentro las tensiones en la región apical medio y cervical del implante.
\end{abstract}

PALABRAS CLAVE: Oseointegración, diseño de los implantes, análisis fotoelástica.

\section{SUMIMARY}

The objective of this study was to analyze the subsequent tensions distribution generated in the photoelastic models after application $7.5 \mathrm{~N}$ vertical loads, in two different dental implant designs. It were selected two different configuration implants, being one conic (Másters Conect Cônico ${ }^{\circledR}$ $4.13 \times 13 \mathrm{~mm}$ ) and the other cylindrical (Titamax Ti Medula ${ }^{\circledR} 3.75 \times 13 \mathrm{~mm}$ ). For the analysis of the

* Estudiante de Doctorado. División de Cirugía Bucal y Maxilofacial. Facultad de Odontología de Piracicaba. Universidad Estadual de Campinas. Brasil.

* Profesor. Departamento de Odontología Integral. Facultad de Medicina. Universidad de La Frontera. Chile y Estudiante de Doctorado. División de Cirugía Bucal y Maxilofacial. Facultad de Odontología de Piracicaba, Universidad Estadual de Campinas. Brasil.

*** Profesor Titular. División de Cirugía Bucal y Maxilofacial. Facultad de Odontología de Piracicaba. Universidad Estadual de Campinas. Brasil. 
tensions distribution it were made two photoelastic resin models with dimensions of $40 \times 10 \mathrm{~mm}$ of base and $30 \mathrm{~mm}$ of height. Each implant was positioned in the model and received vertical loads to $7,5 \mathrm{~N}$, being possible to visualize the isochromatic strips generated in it, through the polariscope. The Másters Conect Cônico ${ }^{\circledR}$ implants, that have long internal hexagon, polished cervical portion, top of spirals rounded, continuous passage of the spirals, distances between these of 0.5 tenth of millimeters, angled spirals and conical apex, presented the concentration of the tensions in the apical region. The cylindrical implants (Titamax Ti Medula ${ }^{\circledR}$ ) with external hexagon, polished cervical portion, with top of the spirals in form of a " $v$ ", continuous passage of spiral, with distance between the spirals of 0,5 tenth of millimeters, angled spirals and rounded apex, presented the distribution of the tensions on apical and cervical regions of the implants. Based on the results of this study it is possible to conclude that: the conical implants configuration concentrated the tensions in apical region, being the cylindrical configuration tensions concentrated on the apical and cervical region of the implants.

KEY WORDS: Osseointegration; Implant Design; Photoelastical analysis.

Fecha de recepción: 8 de enero 2008.

Fecha de aceptación: 16 de enero 2008.

\section{INTRODUCCIÓN}

Una gran evolución dentro de la odontología fue la reposición de dientes naturales perdidos por implantes oseointegrados, principalmente a partir del trabajo de algunos autores (1). Factores de conocimiento sobre la biología de los tejidos adyacentes, perfeccionamiento de materiales biocompatibles, diseño y superficie de los implantes, bien como los aspectos biomecánicos, técnicas quirúrgicas y condiciones de cargas influenciaron directamente el éxito de la oseointegración de los implantes dentales (1).

Un factor importante que afecta el tratamiento con implantes oseointegrados es la transferencia de las fuerzas oclusales para la interfase hueso-implante. La magnitud de esas fuerzas depende mucho del diseño del implante y de sus propiedades mecánicas y estructurales. Esa interfase debe tolerar fuerzas oclusales sin presentar una respuesta adversa a los tejidos adyacentes. Se caracteriza un sistema de implantes por sus macro y microestructuras, propiedades intrínsecas, tipo de conexión implante intermediario, presencia o ausencia de espirales, diseños de espirales, microarquitectura de superficie y composición química.

Los implantes dentales son sometidos a varias magnitudes de fuerzas durante la función masticatoria, por tal motivo éstos acaban transfiriendo las cargas oclusales a los tejidos biológicos. El objetivo de los dise- ños funcionales es transmitir las cargas biomecánicas por medio de una mejor distribución de fuerzas, optimizando la función de las prótesis soportadas por esos implantes (1). Sin embargo no es fácil cuantificar la intensidad de fuerza que puede llevar a la sobrecarga, pues la capacidad ósea individual es variable.

Por tales motivos, estudios de laboratorio son de extrema importancia para analizar situaciones clínicas. Basado en esto el análisis fotoelástico es un método experimental que permite visualizar tensiones y deformaciones a través del polariscopio, entregando información en forma de franjas, tornándose posible determinar la dirección y la intensidad de las tensiones.

El objetivo de este estudio fue analizar la distribución de las tensiones generadas en los modelos fotoelásticos posterior la aplicación de cargas verticales a 7,5 N, en dos diferentes diseños de implantes endoóseos.

\section{MATERIAL Y MÉTODOS}

Los sistemas de implantes utilizados en este estudio fueron: Máster Conect Cônico ${ }^{\circledR} 4,3 \times 13$ mm (Conexão Sistema de Prótesis, São Paulo, SP Brasil), y Titamax Ti Medular ${ }^{\circledR} 3,75 \times 13$ mm (Neodent, São Paulo, SP Brasil) (Tabla 1 y Fig. 1). 


\begin{tabular}{|l|c|c|}
\hline \multicolumn{2}{|c|}{ TABLA 1.- MARCA COIMERCIAL, SISTEMA (FABRICANTE) Y CANTIDAD DE } \\
IMPLANTES UTILIZADOS \\
\hline Marca comercial & Sistema - Fabricante & Cantidad \\
\hline Máster Conect Cônico ${ }^{\circledR}$ & Conexão - Sistema de Prótesis & 01 \\
\hline Titamax Ti Medular ${ }^{\circledR}$ & Neodent - Implante Osseointegrável & 01 \\
\hline
\end{tabular}

\section{ANÁLISIS FOTOELÁSTICO}

\section{a. Polariscopio}

El análisis de la distribución de las tensiones producidas en los modelos fotoelásticos fue realizada a través de imágenes obtenidas del polariscopio circular, este permitió registrar fotográficamente las franjas isocromáticas producidas en el momento de la aplicación de la carga vertical sobre los dos modelos.

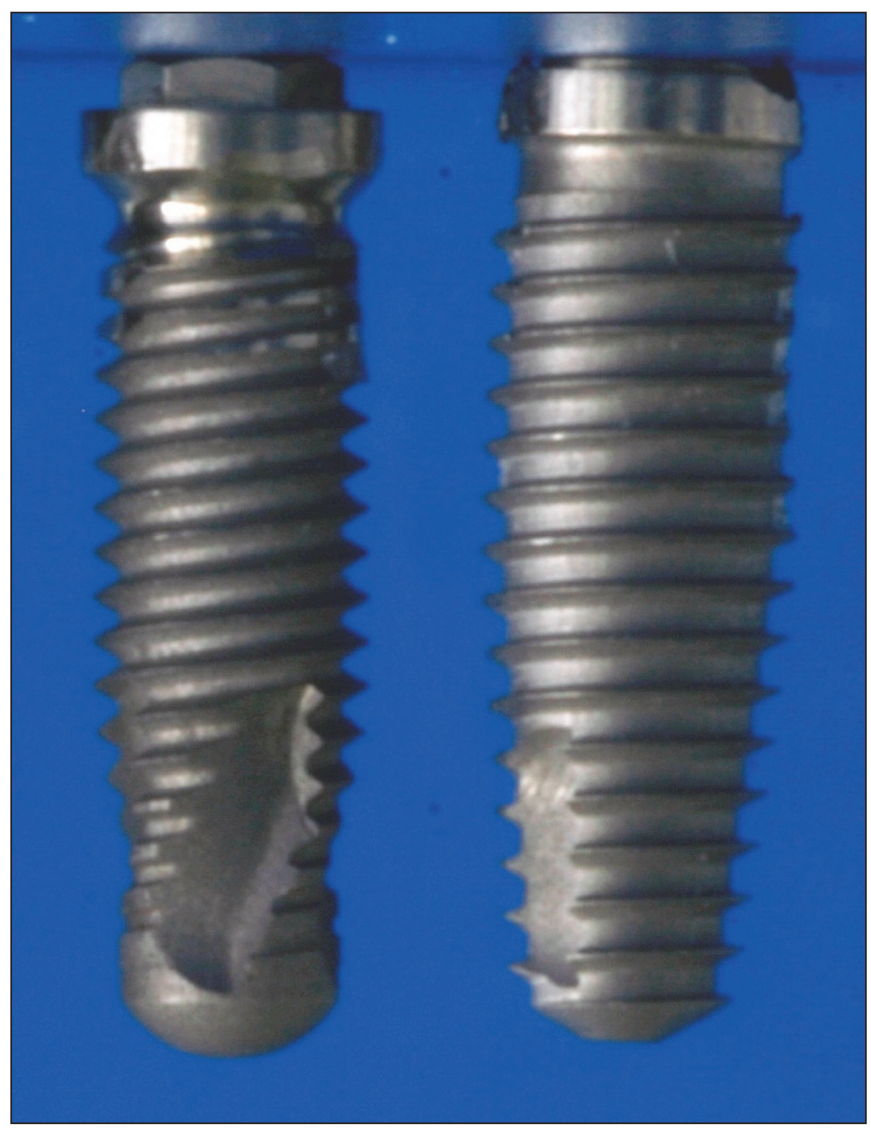

Fig. 1: Morfología de los implantes utilizados en esta investigación: (1) cónico, hexágono interno y (2) cilíndrico, hexágono externo.

\section{b. Modelo fotoelástico}

Dos modelos en acrílico pulido con dimensiones de $40 \times 10 \mathrm{~mm}$ de base y $30 \mathrm{~mm}$ de altura fueron confeccionados y posteriormente duplicados con una silicona de laboratorio (Silibor ${ }^{\circledR}$, Clássico, São Paulo-SP). Una vez duplicados los modelos se posicionaron los diferentes implantes en el interior de la silicona, este presentaba en el medio un dispositivo que permitía mantener en posición vertical y sin movilidad el implante, que seria posteriormente cubierto por la resina fotoelástica.

Siguiendo la proporción de 1:1,20 ml de cada uno de los componentes de la resina fotoelástica, (Araltec ${ }^{\circledR}$ Productos Químicos Ltda. - São Paulo) se introdujeron en un Becker, siendo manipulados con un bastón de vidrio por 10 minutos en movimientos circulares, lento, con la finalidad de obtener una mezcla de coloración homogénea. El recipiente se llevó a una cámara vacuo, en la cual permaneció 15 minutos, con la finalidad de eliminar bolas de aire en el interior de la resina. Una vez obtenido una resina homogénea y sin bolas de aire, esta fue vertida en el interior de la impresión recubriendo todos los espirales del implante, mimetizando la condición biológica de la oseointegración. Posteriormente esa resina permaneció 72 horas en ambiente fresco y aireado para la total polimerización de la resina fotoelástica. Posterior al periodo de polimerización estos fueron retirados de los moldes e inmediatamente fueron colocados los tornillos cobertores (Fig. 2).

\section{c. Ensayo de fotoelasticidad}

En una evaluación fotoelástica preliminar a través del polariscopio, los dos modelos se consideraron libres de tensiones residuales, estando pronto para el inicio del ensayo (Fig. 3). Registros fotográficos fueron realizados en tres momentos, antes durante y posterior a la aplicación de las cargas. Ambos modelos fueron so- 


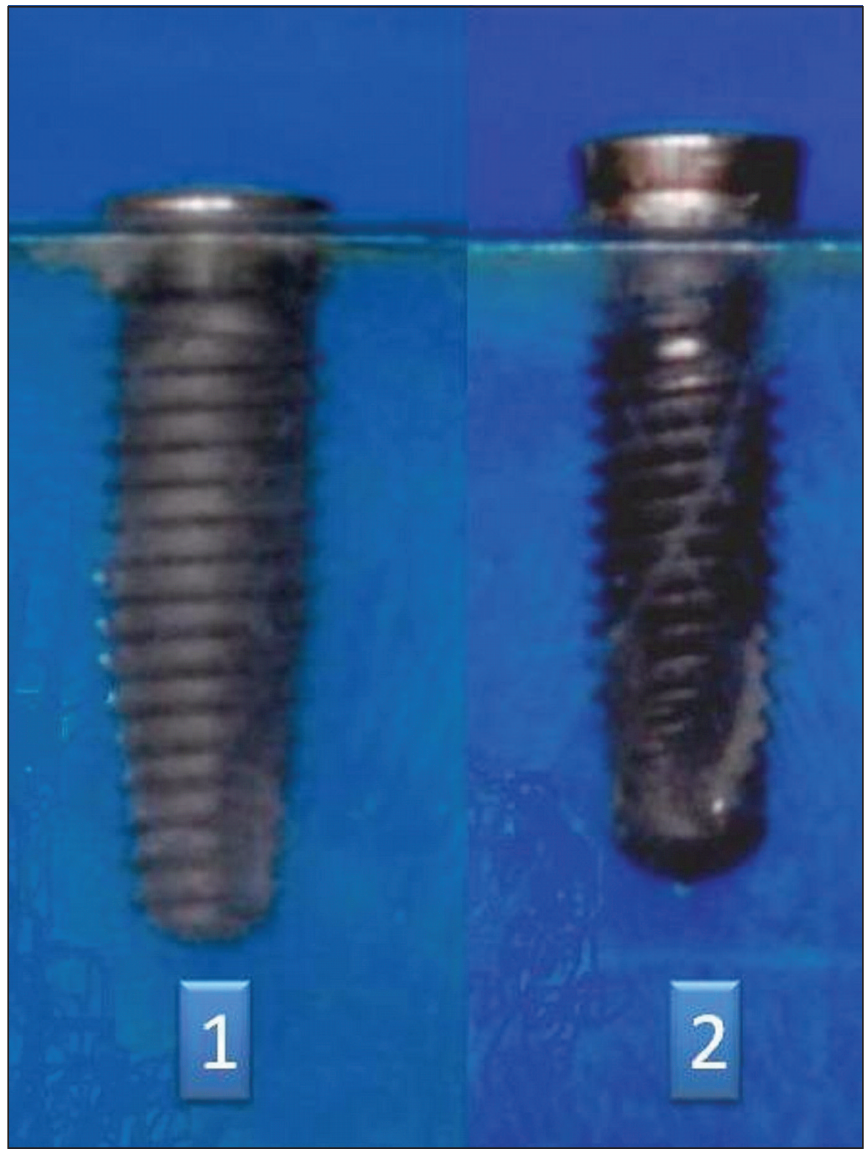

Fig. 2: Implante Titamax Ti Medular ${ }^{\circledR}$ (1) e Implante Máster Conect Cônico ${ }^{\circledR}(2)$ inmerso en resina fotoelástica.

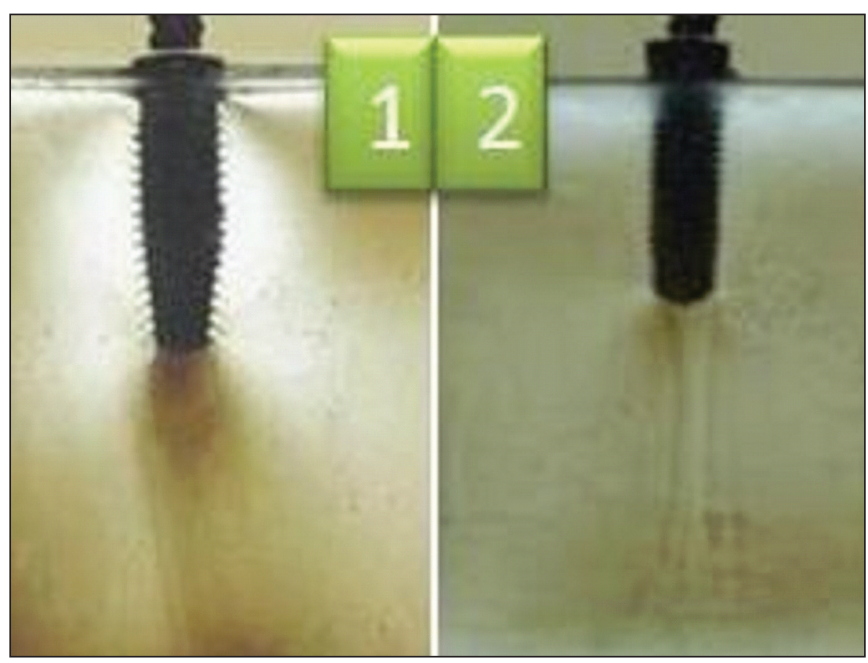

Fig. 3: Implante Implante Máster Conect Cônico ${ }^{\circledR}$ (1) y Titamax Ti Medular ${ }^{\circledR}$ (2) inmerso en resina fotoelástica en estado libre de tensiones residuales. metidos a cargas verticales progresivamente, hasta llegar a $7.5 \mathrm{~N}$. A través del análisis fotoelástico, fue posible evaluar descriptivamente las tensiones generadas a lo largo del implante a medida que la carga era aplicada. Las franjas formadas posterior a la aplicación de cargas, pueden ser cualitativamente observadas a través de una escala de colores utilizada por algunos autores (3) que muestran una presentación de acuerdo con la cantidad de tensiones obtenidas en cada región del cuerpo de prueba.

\section{RESULTADOS}

El implante Máster Conect Cônico ${ }^{\circledR} 4,3 \times 13 \mathrm{~mm}$, con porción cervical pulida de $1 \mathrm{~mm}$ de altura, con tope de las roscas arredondeadas, pasos de las roscas continuas, con distancia entre estas de 0,5 décimas de milímetros, espirales angulados, ápice cónico, y configuración cónica, concentraron las tensiones en la región apical cuando sometidos a carga vertical de 7,5 N (Fig. 4).

El implante Titamax Ti Medula 3,75×13 mm, con porción cervical pulida de $1 \mathrm{~mm}$ de altura, con tope de

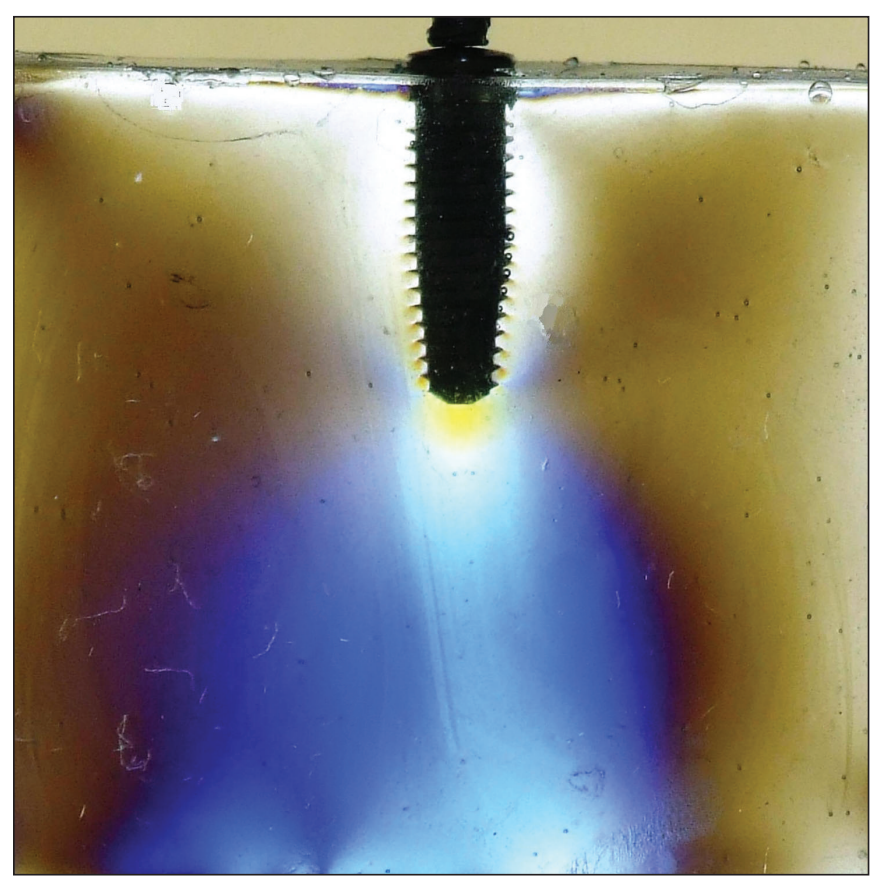

Fig. 4: Concentración de las tensiones en apical del implante cónico de hexágono interno. Se puede observar las franjas isocromáticas. 
las roscas en forma de " $\mathrm{v}$ ", pasos de las roscas continuas, con distancia entre estas de 0,5 décimas de milímetros, espirales angulados, ápice redondeado, y configuración cilíndrica, presentaron distribución de las tensiones en la región apical, medio y cervical del implante cuando sometido a carga vertical a 7,5 N (Fig. 5).

\section{DISCUSIÓN}

A través de este estudio fue posible analizar la distribución de las tensiones en dos diferentes diseños de implantes cuando se sometieron a carga vertical a 7.5 $\mathrm{N}$. El método fotoelástico presenta como ventaja la obtención de informaciones visuales directas sobre el padrón de tensiones que ocurre en un modelo confeccionado con resina fotoelástica posterior a la aplicación de cargas.

Los resultados presentaron diferencias en la distribución de los patrones coloridos, a pesar de ser sometidos a la misma carga, pues el diseño de los implantes es diferente, lo que indicaría una relación del diseño con el padrón de esta distribución de tensiones.

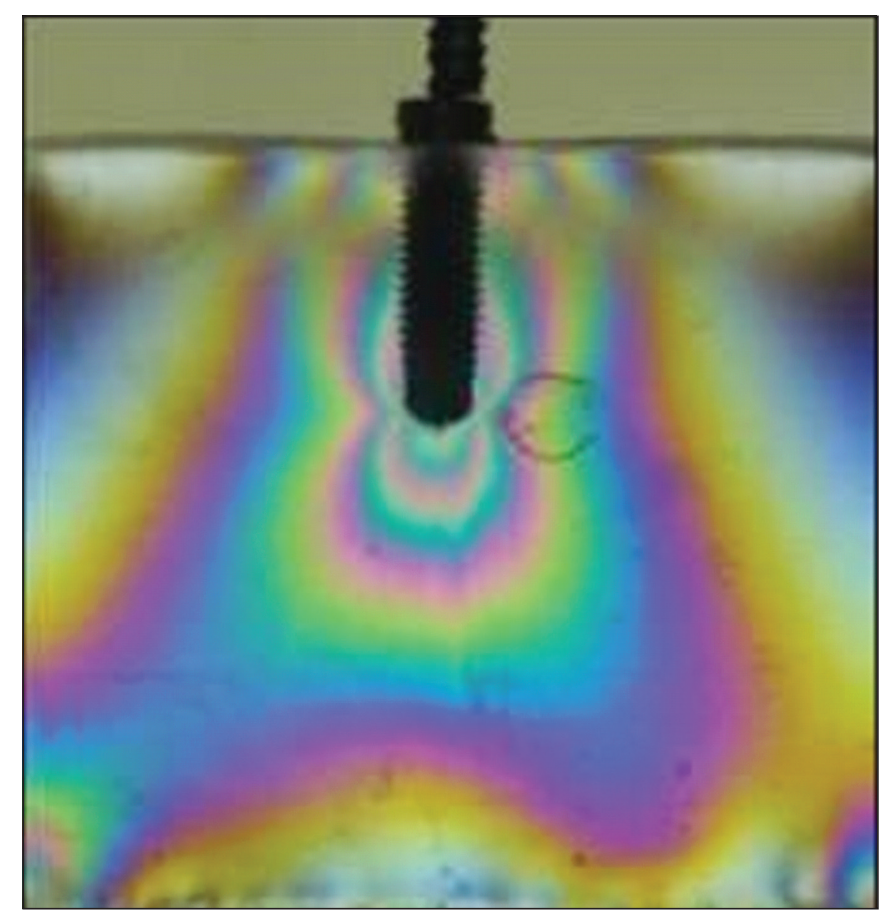

Fig. 5: Concentración de las tensiones en apical medio y cervical del implante cilíndrico de hexágono externo. También es posible observar las franjas isocromáticas.
Al contrario de sólo alcanzar éxito en la oseointegración, existe también una gran preocupación en solucionar con éxito rehabilitaciones sobre implantes en regiones que presentan condiciones desfavorables. Para eso, regiones que presentan defectos en tejidos duros y blandos, necesitan de diseños especializados de implantes. De esa forma las industrias vienen fabricando diferentes diseños y configuraciones de implantes, para que el profesional tenga una gran libertad de escoger el debido implante para cada situación (2).

Los resultados de este estudio muestran que si el objetivo es minimizar las tensiones periimplantarias en la región de la cresta alveolar, la elección clínica más favorable es la de un implante que presente conicidad y hexágono interno largo, evitándose implantes cilíndricos, especialmente en los casos de implantes con carga inmediata y en regiones de hueso con baja calidad, contrariando los resultados presentados por Petrie (3), quien comparo 16 implantes a través de modelos tridimensionales de elementos finitos, verificando mayores tensiones periimplantarias en los implantes cortos y cónicos.

Otros autores $(3,4)$ realizaron estudios relacionados a la comparación del diámetro de los implantes oseointegrados y su distribución de tensiones. Estudios mostraron que los implantes de mayor diámetro presentaron características como menor stress al hueso, debido a la mayor área de superficie implante-hueso. Se observo, además que hay un menor riesgo de fractura del implante debido a la mayor área de metal que circunda en cervical del implante, proporcionando así, mejor apoyo y estabilidad del componente protésico al implante.

Otros autores estudian y comparan implantes con diferentes diseños de roscas y las ventajas en la distribución de las tensiones entre esas diferentes configuraciones (5-7)

Se relataron (5) modificaciones en el diseño y superficie del implante para aumentar el éxito de la oseointegración y proporcionar mejor distribución de cargas en hueso de baja calidad. De esa forma, debido a la función de los implantes en transferir cargas oclusales a los tejidos biológicos, el objetivo de los diseños funcionales es entonces, transferir las cargas biomecánicas a través de una mejor distribución de esas fuerzas, mejorando la función de las prótesis soportadas por los implantes.

Evaluando los dos diseños de implantes utilizados en este estudio, podemos observar que el implante cóni- 
co concentró las tensiones en apical a diferencia del implante cilíndrico que distribuyó las tensiones a lo largo del implante.

\section{CONCLUSIÓN}

Implantes de configuración cónica y hexágono interno largo (Master Conect Cónico 4,13×13 mm) concentro las tensiones en apical sin distribuir a región medio y cervical. Por otra parte, el implante de configuración cilíndrica (Titamax Ti Medula 3,75×13 mm) presentó distribución de tensiones en los tercios apical medio y cervical.

\section{BIBLIOGRAFÍA}

1. Branemark PI, Hansson BO, Adell R, Breine U, Lindstrom J, Hallen O, Ohman A. Osseointegrated dental implants in the treatment of edentulous jaws. Experience from a 10 year period. Scand J Plast Reconstr Surg. 1977;16:1-13.

2. Zadeh HH, Daftary F. Implant designs for the spectrum of esthetic and functional requirements. J Calif Dent Assoc 2004;32(12):1003-10.

3. Petrie CS, Willians JL. Comparative evaluation of implant designs: imfluence of diameter, length, and taper on strains in the alveolar crest. A tree-dimensio- nal finite-element analysis. Clin Oral Implants Res 2005;16(4):486-94.

4. Misch CE. Implant design considerations for the posterior regions of the mouth. Implant Dent 1999; 8 (4):376-86.

5. Steigenga JT, al-Shammari KF, Nociti FH, Misch CE, Wang HL. Dental implant design and its relationship to longterm implant success. Implant Dent 2003; 12(4):306-17.

6. Bumgardner JD, Boring JG, Cooper RC Jr, Gao C, Givaruangsawat S, Gilbert JA et al. Preliminary evaluation of a new dental implant design in canine models. Implant Dent 2000;9:252-60.

7. Itoh $\mathrm{H}, \mathrm{Caputo} A \mathrm{~A}, \mathrm{Kuroe} \mathrm{T}, \mathrm{Nakahara} \mathrm{H}$. Biomechanical comparison of straight and staggered implant placement configurations. Int $J$ Periodontics Restorative Dent 2004;24:47-55.

\section{CORRESPONDENCIA}

Miguel Ángel Jaimes Pérez

Departamento de Diagnóstico Oral

Área de Cirurgia Buco-maxilo-facial

Av. Limeira, 901, Bairro: Areião

CEP: 13414-903. Piracicaba -SP

Fone: (19) 92258022

e-mail:miajape@fop.unicamp.br 